www.jmscr.igmpublication.org

Impact Factor 5.84

Index Copernicus Value: 83.27

ISSN (e)-2347-176x ISSN (p) 2455-0450

crossref DOI: _https://dx.doi.org/10.18535/jmscr/v5i6.216

Journal Of Medical Science And Clinical Research

IGM Publication

An official Publication of IGM Publication

\title{
New Onset Unprovoked Seizures in Elderly: Etiology and Challenges in Management
}

Authors

Dr M.Thangaraj ${ }^{1}$, Dr M.Arun Kumar ${ }^{2}$, Dr K.Balamurali ${ }^{3}$, Dr S.Elangovan ${ }^{4}$

${ }^{1}$ Professor and HOD, ${ }^{2}{ }^{\text {st }}$ Year DM Neurology Resident, ${ }^{3,4}$ Senior Assistant Professor

Department of Neurology, Thanjavur Medical College Thanjavur

Corresponding Author

Dr M.Arun Kumar

$1^{\text {st }}$ Year DM Neurology Resident, Department Of Neurology, Thanjavur Medical College Thanjavur

\begin{abstract}
Contrary to popular belief the incidence of epilepsy and seizures increases in elderly people. New onset epilepsy in elderly often has underlying etiology including cerebrovascular diseases, neurodegenerative disorders, intracranial space occupying lesions and post traumatic epilepsy. An acute symptomatic seizure provoked by metabolic derangement, encephalopathy or meningitis, though commonly seen in elderly patients, can't be termed as unprovoked seizures. Making a proper diagnosis in these patients may be difficult because many times history is not clear. Moreover elderly patients make a heterogeneous group of people. Many of them may be quite healthy while others may have chronic debilitating diseases involving nervous system like dementia, Alzheimer's and Parkinson's disease etc. With improvement in quality of life and availability of advanced health care facilities there is a rise in life expectancy of elderly individuals. This increase in life expectancy has an effect of increase in number of elderly people which consequently means there will be increasing number of patients having newly onset epilepsy beyond the age of 60. Not only the diagnosis is challenging in these patients but also treatment is difficult. Age related physiological changes, presence of co-morbidities, financial constrains and neglect by family members are some of the practical hurdles faced by treating physician while treating these patients. Treatment needs to be carefully selected because many elderly patients are expected to be already on multiple medications due to comorbidities. Anti-epileptic drugs in these patients needs to be chosen keeping in mind Side effects, drug interactions and tolerability of the prescribed drugs We conducted a prospective study comprising of patients who had first episode of unprovoked seizures at or above the age of 60 years. The seizure characteristics, etiology, radiological abnormalities and response to treatment in these patients were studied in detail.
\end{abstract}

Keywords: Unprovoked Seizures, elderly, co-morbid conditions, anti-epileptic drugs.

\section{Introduction}

No age group is immune to seizures. The etiology of seizures differs widely depending upon the age of the patient. With improvement in quality of life, advancement in medical facilities and rise in life expectancy the fastest growing age group with early onset seizures is elderly patients over the age of 60 years ${ }^{[1]}$. Seizures in elderly are usually focal and associated with longer duration of postictal confusion as compared to younger patients. There 
is also more incidence of status epilepticus and the mortality is also higher in this age group ${ }^{[2]}$. The common causes of seizures in elderly include cerebrovascular accidents, encephalopathy and metabolic derangement ${ }^{[3]}$. In many cases the cause cannot be identified. Elderly individuals belong to heterogeneous group of patients with diverse medical problems and pose a significant challenge in diagnosis and management. Moreover the management is complicated by co-existent comorbid conditions and neglect by relatives ${ }^{[4]}$. Another significant challenge in these patients is cognitive problems which makes adherence to medication difficult. Prescribing antiepileptic drugs again poses a challenge because of compromised tolerability as elderly patients are more sensitive to side effects ${ }^{[5]}$.

With an increased life expectancy more and more individuals are living up to their nineties. The elderly patients have traditionally been divided into 3 groups namely young-old (less than 75), middle old (75-84 yrs) and old-old (> $85 \mathrm{yrs}$ ). They can further be divided into groups based on their health status, patients with seizures only, patients with seizures and multiple co-morbid conditions and frail elderly ${ }^{[6]}$. Moreover there is also a huge difference in elderly patients who lives in and cared by large families and those who are neglected or living in old age home. And hence the challenge before a treating physician or neurologist is to modify his approach and treatment taking into account all these factors ${ }^{[7]}$.

The incidence of seizures reported in various studies in elderly individuals (After the age of 60) differs widely. In western countries the incidence is reported to be $125-130 / 100,000$ person-years. In India about 10 million persons have epilepsy. The incidence of epilepsy in elderly individual in India ranges from 1.2 to 11.9 per 1000 population. The actual figures are expected to be far more than what is reported because many times epilepsy in elderly individuals is either under-diagnosed or under-reported $^{[8]}$.

The peculiar thing about seizures in elderly is that many times first episode of unprovoked seizures in these individuals presents as status epilepticus.
The annual incidence of status epilepticus in elderly individuals is reported to be around $85 / 100,000$ persons which is far more than the incidence of status epilepticus in young population. The difficulties in management, morbidity and mortality increases as the age advances ${ }^{[9]}$.

Elderly individuals usually presents with focal seizures but rarely they may present with generalised, absence or myoclonic seizures. Despite varied etiologies the clinical features and response to treatment is usually similar and most of these elderly individuals respond to AEDs fairly uniformly and surgical treatment for refractory surgery is generally not needed.

Despite growing incidence of unprovoked seizures, epilepsy and status epilepticus in elderly individuals there is surprisingly little research have been done ${ }^{[10]}$.

We conducted this study to determine seizure characteristics, etiology, radiological abnormalities and response to treatment in patients with unprovoked seizure at and above the age of 60 .

\section{Materials and Methods}

This study was conducted at government Thanjavur medical college hospital, Thanjavur, Tamil Nadu. In this study 52 patients were studied. All patients at the age of 60 and above with history of unprovoked seizure were included into the study. Their detailed history was taken. Thorough Clinical examination \& investigations were done. Physical examination was done with a special emphasis on examination of neurological and cardiovascular system. Routine investigations like complete blood count, Liver function tests, blood urea and serum creatinine and random blood sugar was done in all cases. An electrocardiogram or chest $\mathrm{x}$-ray was done in selected patients depending upon signs and symptoms. CT scan was done in all patients and Magnetic resonance imaging was done in patients in whom either CT brain was inconclusive or further evaluation was suggested. Electroencephalogram was not done routinely because it is neither sensitive nor specific in elderly patients with epilepsy. Moreover absence of epileptiform 


\section{JMSCR Vol||05||Issue||06||Page 24136-24143||June}

activity doesn't rule out seizure disorder in these patients.

\section{Inclusion Criteria}

1. Unprovoked seizures at and above the age of 60 .

\section{Exclusion Criteria}

1. All provoked seizures

2. Remote symptomatic seizures.

3. Known epileptic patients who are taking AEDs before the age of 60 .

\section{Observations and Results}

52 Patients of age $60 \mathrm{yrs}$ or above presenting with unprovoked seizures in a tertiary care hospital were enrolled in this study. Out of 52 patients enrolled in this study 34 patients were males and 18 patients were females with a male to female ratio of 1: 0.52 (Figure 1).

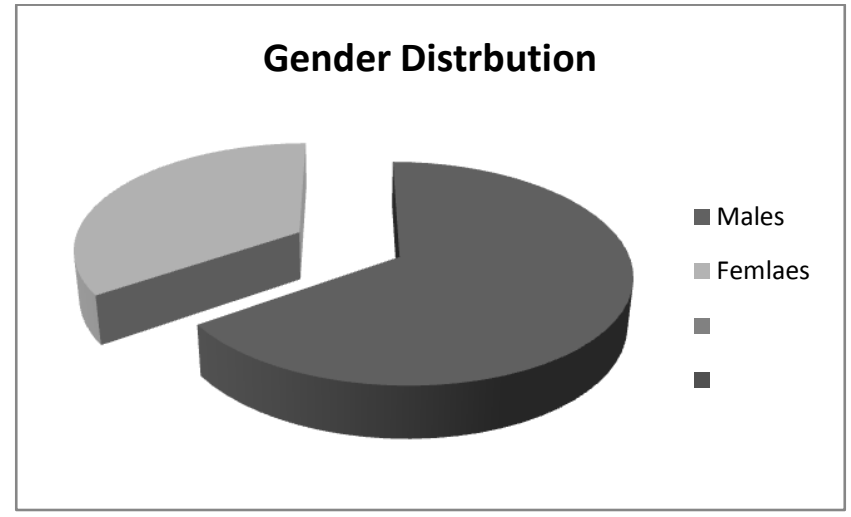

Fig 1: Gender distribution of the studied cases. The patients were further divided on the basis of their age. Most of the patients belonged to age group of 60-65 years (44.23\%) followed by age group of 65-70 (23.08\%),70-75(11.54\%), 75$80(11.54 \%)$ and above the age of 80 years (9.62\%) (Figure 2).

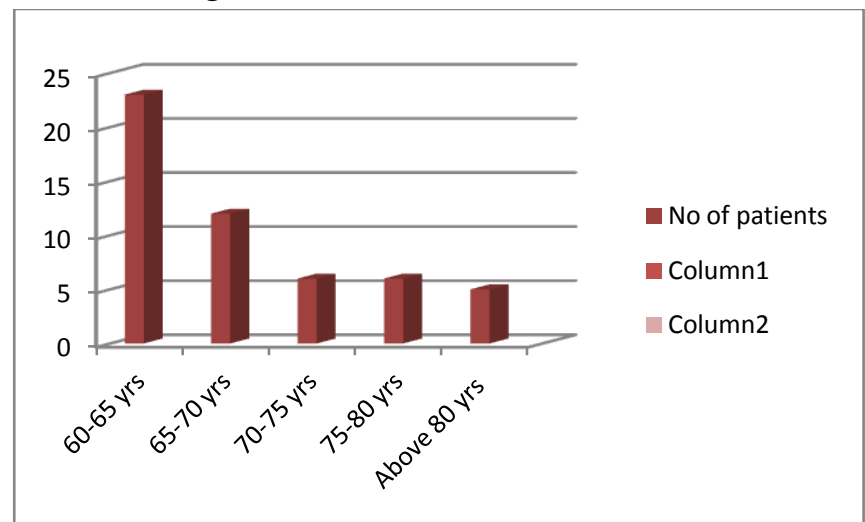

Figure 2: Distribution of patients on the basis of age.
The patients were further subdivided on the basis of whether they belong to rural or urban areas. On the basis of this distribution 19 (36.54\%) patients were found to be belonging to rural areas whereas $33(63.46 \%)$ patients belonged to urban areas. This distribution was important as to know whether there was any undue delay in reaching to hospital after first episode of unprovoked seizures (Figure 3).

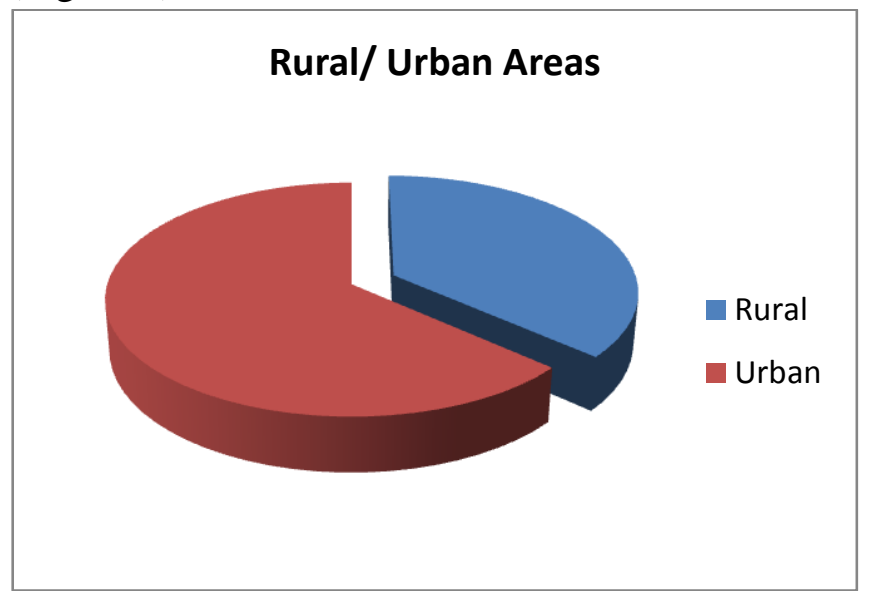

Figure 3: distribution on the basis of whether they belong to rural or urban areas.

The type of seizures was noted in all the patients. The most common type of seizures observed in cases were generalized 42/52 (80.77\%) followed by focal seizures with secondary generalization $5 / 52(9.62 \%)$, complex partial seizures were seen in $3 / 52(5.77 \%)$. Least common type of seizures found during this study was focal seizures which were seen in $2 / 52$ patients (3.85\%) (Figure 4$)$.

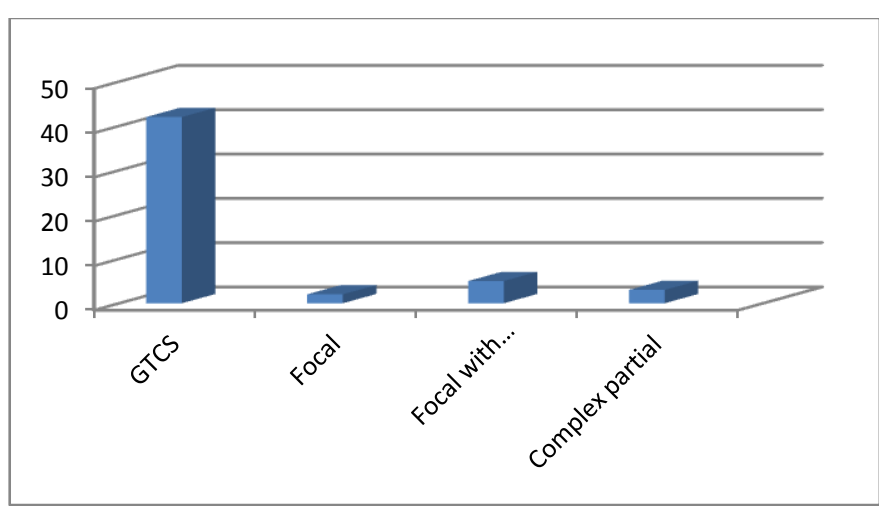

Figure 4: Distribution of the patients on the basis of type of seizures.

All the patients underwent brain imaging in the form of computerized tomography (CT) scan of the brain. Computerized tomography Scan (CT scan) was done in all the patients presenting with 
unprovoked seizures. Most of the patient underwent non-contrast enhanced CT. Contrast studies were done if there was any suspicion during plain CT scan. The time interval between onset of alseizures and CT scanning ranged from 1- 15 days depending upon the time when patient was first brought to hospital, general condition of the patient and willingness of patients or relatives to undergo imaging. Most of the patients enrolled in our study had a normal CT Brain 28/52 $(53.85 \%)$. The most common abnormality found in the CT brain was age related cerebral atrophy which was seen in $12 / 52$ patients $(23.08 \%)$. Other less common abnormalities found in CT brain were Gliosis (7/52), infarcts (4/52) and granulomas/focal lesions (1/52).

Table 1: CT scan abnormalities in studied cases

\begin{tabular}{|c|c|c|}
\hline CT Scan Findings & $\underset{\text { patients }}{\text { No of }}$ & Percentage \\
\hline Normal & 28 & $53.85 \%$ \\
\hline $\begin{array}{l}\text { Age related cerebral } \\
\text { atrophy }\end{array}$ & 12 & $23.08 \%$ \\
\hline Gliosis & 7 & $13.46 \%$ \\
\hline Infarcts & 4 & $7.69 \%$ \\
\hline $\begin{array}{l}\text { Granuloma/ Focal } \\
\text { lesion }\end{array}$ & 1 & $1.92 \%$ \\
\hline Total & 52 & $100 \%$ \\
\hline
\end{tabular}

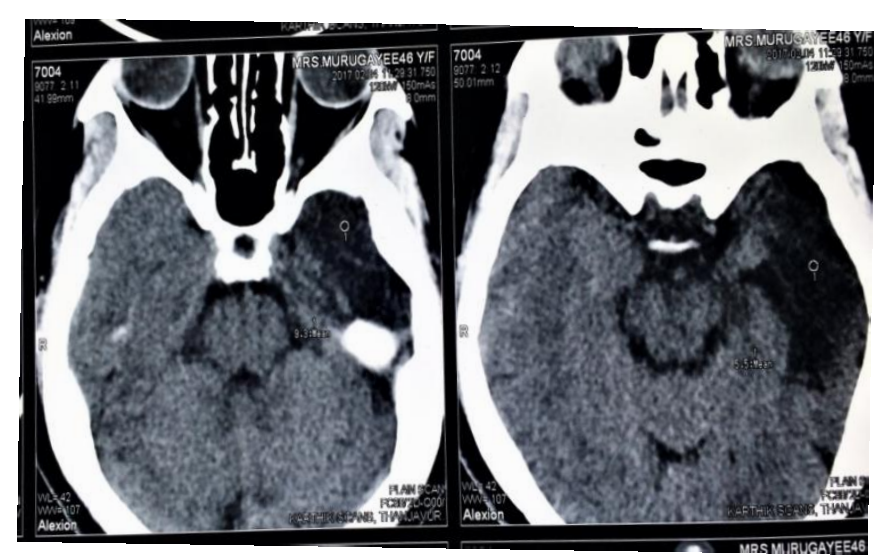

Figure 5: CT Brain showing subacute left temporal lobe infarct. The patient presented with focal seizures involving right side of the body.

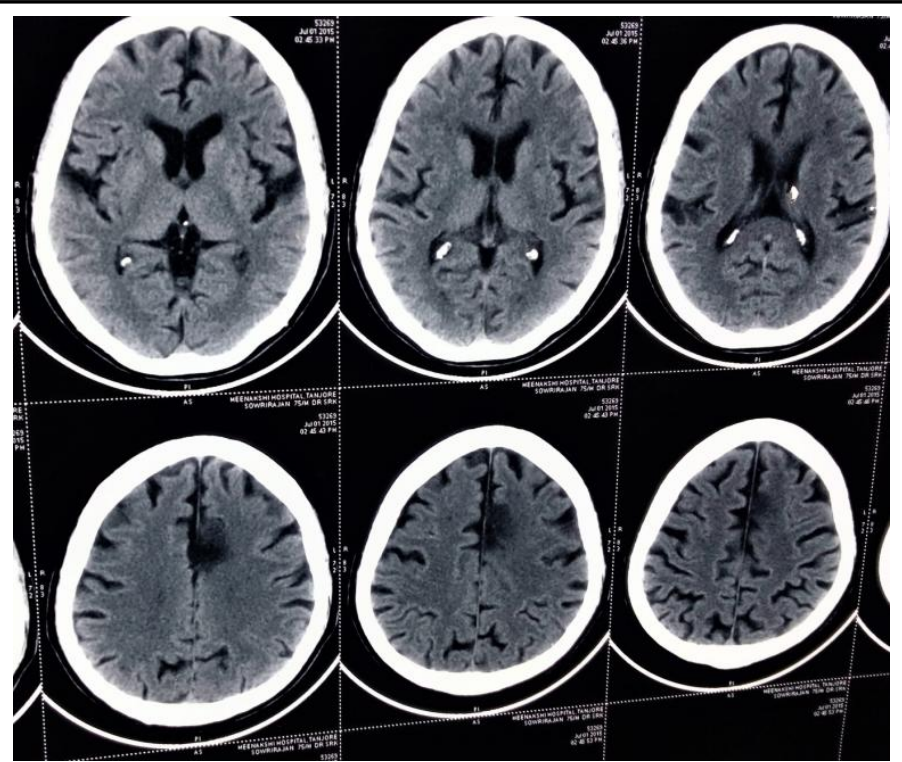

Figure 6: CT Brain showing chronic Anterior Cerebral artery territory infarct.

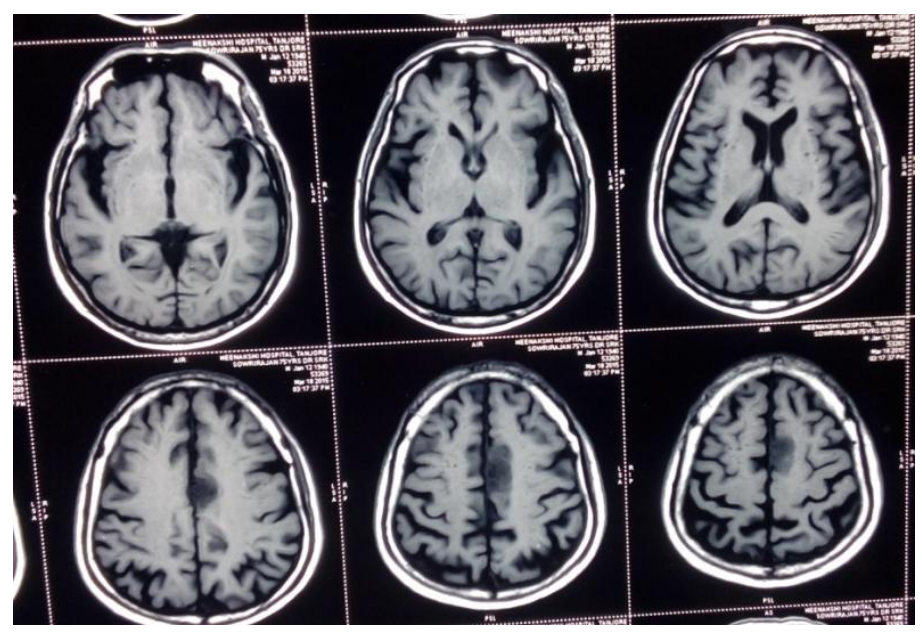

Figure 7: MRI of same patient showing hypointense signals on $\mathrm{T} 1$ weighted images in anterior cerebral artery area suggestive of infarct.

MRI was done in 28 patients on the basis of equivocal findings on CT brain. The other factors which were taken in to consideration while ordering MRI brain were clinical indications, feasibility, and affordability. Out of the 28 patients who underwent MRI scans 8 had a normal MRI (28.57\%) while cortical atrophy and infarcts were found in $6 / 28$ patients each $(21.42 \%)$. While less common finding were small vessel disease (14.28\%), white matter abnormalities (7.14\%), encephalomalacia (3.57\%) and focal lesions/granuloma (3.57\%). 
Table 2: MRI findings in the studied cases

\begin{tabular}{|lcc|}
\hline MRI Findings & $\begin{array}{c}\text { No of } \\
\text { patients }\end{array}$ & Percentage \\
\hline Normal & 8 & $28.57 \%$ \\
\hline Cortical Atrophy & 6 & $21.42 \%$ \\
\hline Infarcts & 6 & $21.42 \%$ \\
\hline Small Vessel disease & 4 & $14.28 \%$ \\
\hline $\begin{array}{l}\text { White } \\
\text { abnormalities matter }\end{array}$ & 2 & $7.14 \%$ \\
\hline Encephalomalacia & 1 & $3.57 \%$ \\
\hline focal lesions/granuloma & 1 & $3.57 \%$ \\
\hline
\end{tabular}

EEG was done in 28 patients depending upon seizure type and whether patient was suitable enough to follow the instructions. It was not done in all patients because in elderly patients with new onset unprovoked seizures EEG is neither specific nor sensitive. Moreover in most of the cases interictal EEG is expected to be normal and hence a normal EEG does not rule out epileptogenic focus. Out of 28 patients who underwent EEG 27 patients had normal EEG and only 1 patient $(3.57 \%)$ had abnormalities in EEG.

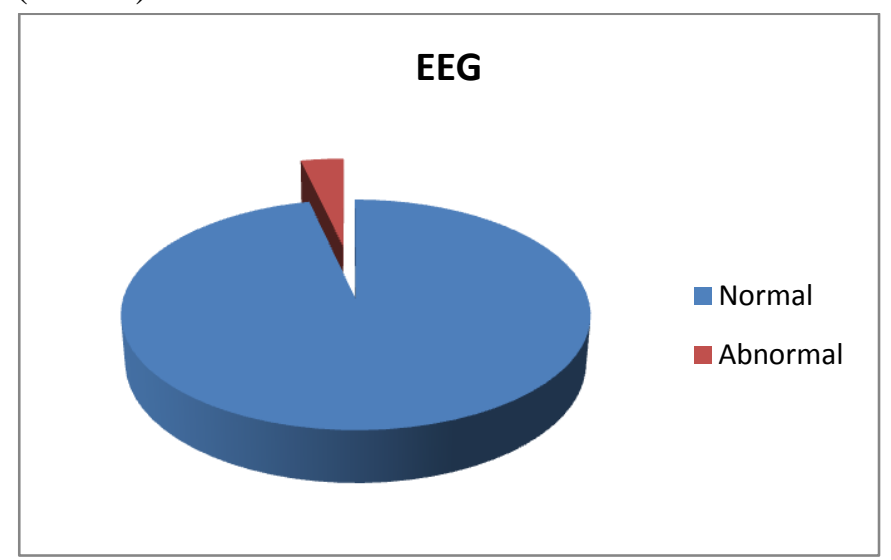

Figure 8: Distribution of normal and abnormal EEG in studied cases.

After stabilization of patients and initial IV doses of anticonvulsants like diazepam, lorazepam and leviteracetam most patients were put on regular anti epileptic drugs. Most patients responded to oral administration of phenytoin sodium. 46/52 patients responded to monotherapy with phenytoin sodium. While Alternative antiepileptic drugs like carbamazepine and sodium valproate were given to 1 patient each. Combination of 2 anti epileptic drugs like Leviteracetam and sodium valproate, Leviteracetam and phenobarbitone, carbamazepine and leviteracetam or phenytoin and carbamazepine was required in 3 patients (7.69\%).

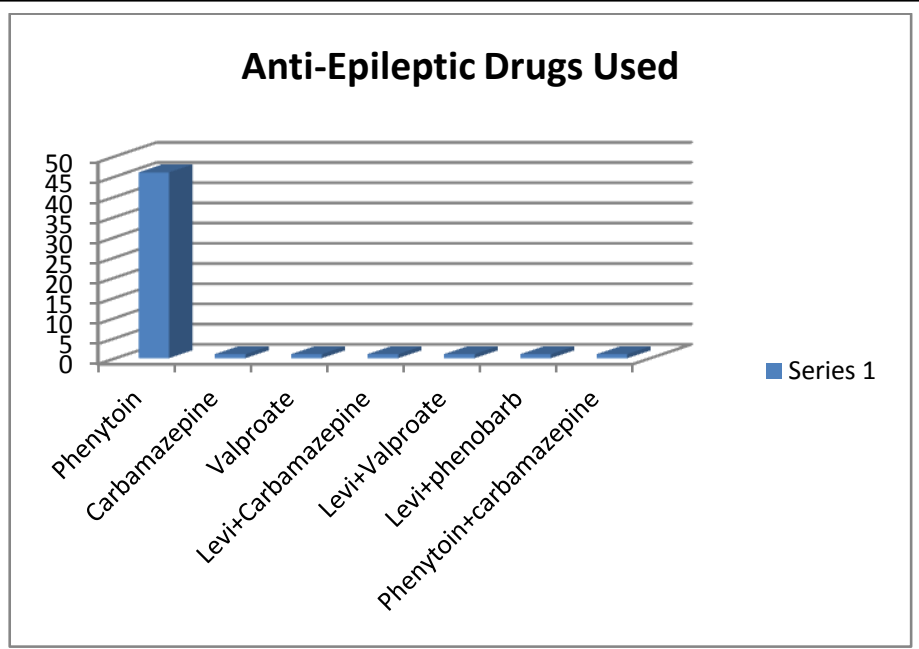

Figure 9: Antiepileptic Drugs Used In studied cases.

\section{Discussion}

As the population of elderly patients ( $>60$ years) increases due to increased life expectancy, these patients constitute the fastest growing age group to have unprovoked seizures. The etiology, clinical features, prognosis, management and complications peculiar to patients in this age group is quite different from those adults who develop seizures at a younger age ${ }^{[11]}$. As the age advances there is a gradual increase in the incidence of new onset unprovoked seizures. With increasing percentage of elderly individuals these numbers are expected to further increase. Moreover the true incidence and prevalence of epilepsy in the elderly patients may in fact be much higher than the numbers quoted above because of difficulties identifying seizures and diagnosing epilepsy ${ }^{[12]}$. Also some of the elderly patients are neglected by their family members and may seek medical attention very late. The incidence of Status epilepticus at first presentation is also more common in elderly patients than their younger counterparts. Because of this there are higher mortality rates due to seizures in elderly In general the mortality in the elderly is around 35\%, but increases to almost $50 \%$ in patients older than 80 years. Status epilepticus is also responsible for prolonged duration of hospitalization, need for artificial ventilation, ventilator associated pneumonias, morbidity and mortality ${ }^{[13]}$. 
Seizures in elderly are usually not associated with aura and automatism. Seizures may present as generalised tonic clonic movements of the body, focal tightening of limbs, facial involvement, staring look and impairment of consciousness ${ }^{[14]}$. Absence seizures are uncommon in elderly patients. The post-ictal phase in these patients is usually prolonged ranging from hours to many days. As stroke is one of the common causes of seizures in elderly extratemporal lobe localization is frequent in elderly patients. In one study seizures in elderly were subdivided on the basis of etiology into 4 groups: idiopathic, secondary hippocampal sclerosis, definite limbic encephalitis and MRI-defined possible limbic encephalitis ${ }^{[15]}$. Common etiological causes of seizures in elderly found out in various studies conducted in developing as well as developed countries include stroke, trauma, space occupying lesions and Alzheimer's disease. Though not subject of these study acute symptomatic seizures can arise from drugs toxicity, metabolic derangements, electrolyte imbalance, meningitis, encephalitis, acute stroke, and head injury ${ }^{[16]}$.

Elderly patients who present with unprovoked seizure after 60 years of age are more likely to develop recurrence than younger adults. If these unprovoked seizures recur the diagnosis of epilepsy is confirmed. 2 or more episodes of unprovoked seizures are classified as remote symptomatic epilepsy if the cause of these unprovoked seizures is identified. This is possible in more than $50 \%$ of the patients. Stroke or a history of stroke not only predisposes elderly patients to develop seizures but also occurrence of unprovoked seizures may increase the risk of subsequent development of stroke in elderly individuals ${ }^{[17]}$.

Another important risk factor for occurrence of seizures in elderly is Alzheimer's disease and other dementias. There I approximately 5-10 fold in the risk of epilepsy in these individuals.

The presentation of seizures is less specific in these individuals. Since many elderly individuals may also have dementia they may not be able to give proper history. In those instances the account of eye witness is invaluable. Many a times these patients are neglected and seizures especially those of partial variety are often overlooked by patient and family members. Moreover in many patients there is a significant delay in seeking medical advice due to neglect by other family members ${ }^{[18]}$.

Detailed and meticulous physical examination of these patients must be carried out with a special emphasis on neurological and cardiovascular examination. Any predisposing finding like presence of murmur, dementia, abnormal movements or focal neurological deficits should be looked for ${ }^{[19]}$. Routine investigations like complete blood count, Liver function tests, renal function tests and random blood sugar should be done in all cases. Chest X-ray, Electrocardiogram and $2 \mathrm{D}$-Echo should be done if there is relevant history or abnormality on physical examination. CT brain should be done in all patients ${ }^{[20]}$. Since MRI is costly and difficult to do in elderly individuals for whom it may be difficult $t$ remain still for a prolonged period of time it may be done in selected patients in whom CT findings are equivocal $^{[21]}$.

Electroencephalography is neither sensitive nor specific in elderly individuals with unprovoked seizures. Moreover false positive results due to artifacts and false negative results in interictal period are quite common ${ }^{[22]}$. EEG must always be read in context with history and clinical examination. In doubtful cases video electroencephalographic monitoring can be done ${ }^{[23]}$.

Antiepileptic drug treatment after first episode of unprovoked seizures is somewhat controversial. While immediate treatment after a first unprovoked seizure may not improve the long term remission rate it may be considered if there is an abnormal EEG, neuroimaging abnormalities or in cases where etiological factors are known to cause recurrent seizures ${ }^{[24]}$. The decision of whether or not to start antiepileptic drugs should be taken after taking into consideration the risk and benefits of starting such a treatment as once started patients are expected to remain on AED for the rest of their lives. Majority of the patients 
respond to monotherapy i.e. phenytoin or phenobarbitone. Alternative drugs like leviteracetam or valproate may be used in other patients. Some patients may need combination therapy consisting of leviteracetam and carbamazepine, leviteracetam and phenobarbitone or phenytoin and carbamazepine ${ }^{[25] \text {. }}$

\section{Conclusion}

Elderly patients form the fastest growing group of individuals developing unprovoked seizures. With improving health care facilities and increasing life expectancy the number of such individuals are expected to rapidly grow. The etiology, presentation and management of these individuals is different from young adults. Special consideration about drug interactions and toxicity is essential while prescribing antiepileptic drugs in these individuals.

\section{Conflict of Interest: None}

\section{References}

1. Brodie MJ, Kwan P . Epilepsy in elderly people. BMJ. 2005 Dec 3; 331(7528):1317-22.

2. DeLorenzo RJ, Hauser WA, Towne AR, Boggs JG, Pellock JM, Penberthy L, Garnett L, Fortner CA, Ko D, A prospective, population-based epidemiologic study of status epilepticus in Richmond, Virginia. Neurology. 1996 Apr; 46(4):1029-35.

3. Liu S, Yu W, Lü Y. The causes of newonset epilepsy and seizures in the elderly. Neuropsychiatric Disease and Treatment. 2016;12:1425-1434.

4. Feinstein AR. The pre-therapeutic classification of co-morbidity in chronic disease. J Chronic Dis. 1970 Dec; 23(7):455-68.

5. Beghi E. Efficacy and tolerability of the new antiepileptic drugs: comparison of two recent guidelines. Lancet Neurol. 2004 Oct;3(10):618-21.
6. Acharya JN, Acharya VJ. Epilepsy in the elderly: Special considerations and challenges. Annals of Indian Academy of Neurology. 2014;17(Suppl 1):S18-S26.

7. Haut SR, Katz M, Masur J, Lipton RB. Seizures in the elderly: Impact on mental status, mood and sleep. Epilepsy \& behavior : E\&B. 2009;14(3):540-544.

8. Olafsson E, Ludvigsson P, Gudmundsson G, Hesdorffer D, Kjartansson O, Hauser WA.

9. Incidence of unprovoked seizures and epilepsy in Iceland and assessment of the epilepsy syndrome classification: a prospective study. Lancet Neurol. 2005 Oct; 4(10):627-34.

10. Mauricio EA, Freeman WD. Status epilepticus in the elderly: differential diagnosis and treatment. Neuropsychiatric Disease and Treatment. 2011;7:161-166.

11. Hauser WA. Epidemiology of epilepsy and seizures in the elderly. In: Rowan AJ, Ramsay ER, editors. Epilepsy in the Elderly. Boston: Butterworth Heinemann; 1997. pp. 7-18

12. Adachi N, Kanemoto K, Muramatsu R, et al. Intellectual prognosis of status epilepticus in adult epilepsy patients: analysis with Wechsler Adult Intelligence Scalerevised. Epilepsia. 2005;46(9):1502-1509.

13. Leppik IE, Birnbaum AK. Epilepsy in the elderly. Ann N $\quad$ Y Acad Sci. 2010;1184:208-224.

14. Logroscino G, Hesdorffer DC, Cascino GD, Annegers JF, Bagiella E, Hauser WA. Long-term mortality after a first episode of status

epilepticus. Neurology. 2002;58:537-541.

15. Kellinghaus C, Loddenkemper T, Dinner DS, Lachhwani D, Lüders HO. Seizure semiology in the elderly: a video analysis. Epilepsia. 2004 Mar;45(3):263-7.

16. Urbach H, Schramm J, Soeder BM, Becker AJ, Voltz R, Vincent A, Elger CE, Bien $\mathrm{CG}$, . Limbic encephalitis as a precipitating 
event in adult-onset temporal lobe epilepsy. Neurology. 2007 Sep 18; 69(12):1236-44.

17. Pandis D, Scarmeas N. Seizures in Alzheimer Disease: Clinical and Epidemiological Data. Epilepsy Currents. 2012;12(5):184-187.

18. Asconapé JJ, Penry JK. Poststroke seizures in the elderly. Clin Geriatr Med. 1991 Aug;7(3):483-92. Review. PubMed

19. Hermann B, Seidenberg M, Sager M, et al. Growing old with epilepsy: The neglected issue of cognitive and brain health in aging and elder persons with chronic epilepsy. Epilepsia. 2008;49(5):731-740.

20. Tian H-J, Wang X-Q, Shi X-B, Lang S-Y. Evaluation of clinical features of elderly epilepsy in China. International Journal of Clinical and Experimental Medicine. 2015;8(2):2399-2404.

21. Sinha S, Satishchandra P, Kalband BR, Bharath RD, Thennarasu K. Neuroimaging observations in a cohort of elderly manifesting with new onset seizures: Experience from a university hospital. Annals of Indian Academy of Neurology. 2012;15(4):273-280.

22. Kesavadas C, Thomas B. Clinical applications of functional MRI in epilepsy. The Indian Journal of Radiology \& Imaging. 2008;18(3):210-217.

23. Maganti RK, Rutecki P. EEG and epilepsy monitoring. Continuum (Minneap Minn). 2013 Jun;19(3 Epilepsy):598-622.

24. Pandian JD, Cascino GD, So EL, Manno E, Fulgham JR. Digital videoelectroencephalographic monitoring in the neurological-neurosurgical intensive care unit: clinical features and outcome. Arch Neurol. 2004 Jul;61(7):1090-4.

25. Poza JJ. Management of epilepsy in the elderly. Neuropsychiatric Disease and Treatment. 2007;3(6):723-728.
26. Jankovic SM, Dostic M. Choice of antiepileptic drugs for the elderly: possible drug interactions and adverse effects. Expert Opin Drug Metab Toxicol. 2012 Jan;8(1):81-91. 